\title{
Social Marketing. Ein hilfreicher Weg für die Kirche?
}

\author{
von Thomas Schierl
}

Angesichts sich häufender Austritte von Mitgliedern der beiden großen christlichen Kirchen stellt sich - zumindest für denjenigen, der nicht unbedingt der Meinung ist, es handle sich hierbei um ein vielleicht sogar notwendiges "Gesundschrumpfen" - die Frage, ob Werbung ein geeignetes Mittel zur Verlangsamung, zum Stoppen oder sogar zum Umkehren dieses Prozesses sein könnte und wenn ja, wie eine Werbung für Kirche aussehen müßte bzw. wie sie nicht aussehen dürfte.

\section{Kirche und Werbung}

Werbung für die Kirche ist früher wie heute und innerhalb wie außerhalb der Kirche ein Reizthema. Auch wenn Pastor Paul Hohenstein fortschrittlicherweise schon 1921 in der Zeitschrift "Kultur der Reklame“ über Vorzüge und Notwendigkeit einer kirchlichen Werbetätigkeit, Verkaufsförderung und Vertriebsstruktur schrieb ${ }^{1}$, so dominierten innerhalb der Kirche doch immer eher die Vorbehalte gegen eine kirchliche Werbung. Unversöhnlich standen sich die Positionen der Befürworter und der Gegner, denen Altruismus und Marketing unvereinbar schienen, gegenüber. Die in den $50 \mathrm{er}$ Jahren beginnende und sehr emotional geführte Diskussion über manipulative Werbung, ausgelöst oder zumindest verstärkt durch Vance Packards "Die geheimen Verführer", verhärtete die Front der Gegner kirchlicher Werbung noch mehr ${ }^{2}$. Diese Distanz zur Werbung ist allerdings nicht

Dr. Thomas Schierl ist Mitarbeiter am Rhein-Ruhr Institut für Sozialforschung und Politikberatung (RISP).

1 Werbung für den lieben Gott, in: InSight Kommunikation (1994) Nr. 6, 26 (o. V.).

2 H. Tremel, Einleitung, in: ders. (Hg.); Öffentlichkeitsarbeit der Kirche, Frankfurt ?1990, 7-24, 8ff.

Berührungsängste mit Werbung gab es eigentlich nie im Bereich der karitativen bzw. diakonischen Werbung, die zu einem großen Teil dazu dient, die Spendenbereitschaft bei der Bevölkerung zu erhöhen. In diesem Bereich wurde das Werbewissen zumindest viel stärker als bei der kirchlichen Werbung professionalisiert (vgl. hierzu auch Helmut E. Thormann, Öfentlichkeitsarbeit im diakonischen Unternehmen - das Beispiel Hephata, in: ebd., 159-174). „Am stärksten ausgebaut ist die Öffentlichkeitsarbeit der Diakonie und der Entwicklungspolitik. Innerhalb des Spendenwesens arbeitet kirchliche Werbung und Offentlichkeitsarbeit seit langem professionell. Zu denken geben sollte es, daß dort, wo Öffentlichkeitsarbeit 
nur alleine innerhalb der klerikalen Berufsgruppe, sondern allgemein in Sozialberufen häufig anzutreffen ${ }^{3}$.

Aber nicht nur der Kirche, selbst ausgebufften Werbeprofis kann es scheinbar schon einmal ein wenig unbehaglich werden beim Thema Kirche und Werbung. So kommentierte das Werbe-Fachblatt "new business" in seiner ,Ketzer-Ecke' den neuen Werbeauftritt der evangelischen Stadtkirche Köln mit einer äußerst bissigen Glosse unter dem Titel "Warum nicht mal Gott vermarkten?" in der sarkastisch Sponsorships durch Luftfahrtgesellschaften (Denn "wer ist dem Himmel näher als die Lufthansa?") ebenso wie Unterbrecherwerbung während der Messe vorgeschlagen wurden 4 .

Innerhalb der Kirche resultieren bei näherem Betrachten viele Vorbehalte (eventuell auch Befürwortungen) vornehmlich aus mangelndem Wissen über Werbung und Mißverstehen von dem, was Werbung eigentlich ist. Schon Mitte der $60 \mathrm{er}$ Jahre diagnostizierte und beklagte Josef Bennemann diesen Mangel klar und deutlich: "Wenn in kirchlichen Kreisen über Werbung gesprochen wird, so steht im allgemeinen die kritische Beurteilung im Vordergrund. Nicht immer liegt dem Urteil eine genügende Kenntnis der Werbung, ihrer Methoden und ihrer Aufgaben zugrunde. (...) Und schließlich geht es um den Einsatz ihrer Methoden für kirchliche Zwecke. Die Meinungen zu dieser Frage stehen sich noch häufig diametral gegenüber. Fachleute der Werbung und Fachleute der Seelsorge sprechen eine verschiedene Sprache. Die Mißverständnisse sind oft groß. Deshalb ist es nötig, das gegenseitige Verstehen durch besseres Wissen voneinander zu vergrößern" 5 . Aber die Schuld für das Mißverstehen der Werbefachsprache liegt nicht bei den Fachleuten der Seelsorge, sondern bei denen der Werbung. Denn deren fachlicher Begrifflichkeit mangelt es an genügender Trennschärfe. Begriffe wie Reklame, Werbung, Öffentlichkeitsarbeit, Public Relations, Public Affairs, Human Relations, Marketing, Social Marketing, Marketing für Nonprofit-Organisationen usw. werden selbst von Werbefachleuten ganz uneinheitlich und oftmals sogar synonym gebraucht, was auch daran liegt, daß es bei weitem noch keine ausgearbeitete wissenschaftliche Theorie der Öffentlichkeitsarbeit gibt ${ }^{6}$, genausowenig

am weitesten ausgebaut ist, die kirchliche Arbeit in der Bevölkerung an Image gewonnen hat. Das ist neben der Diakonie auch in der Entwicklungspolitik der Fall." (Gemeinschaftswerk der Evangelischen Publizistik (Hg.), Medien und Kirche. Einführung in das Schwerpunktthema der Synode der Evangelischen Kirche in Deutschland vom 1. bis 6. November 1992 in Suhl, o.O. 1992, 63)

3 B. Clemens, ... jeder Tag zăhlt, in: InSight Kommunikation (1993) Nr. 8, 19-21, 20.

4 New business (1992) Nr. 41, 17.

5 J. Bennemann, Kirche und Werbung, Osnabrück 1966, 9.

6 H. Tremel (Anm. 2), $11 \mathrm{ff}$. 
wie ein einheitliches Theorie- oder wenigstens Terminologieverständnis, welches der Praxis zugrundeliegt.

Diese Begriffsunsicherheit führt u.a. wiederum zu falschen oder unsinnigen Begründungen für die Befürwortung genauso wie für die Ablehnung von kirchlicher Werbung. Die Befürworter beispielsweise sehen Werbung als wesensimmanenten Zug der Kirche an. Denn der Gott der Christen ist für diese ein werbender Gott, dessen Bemühen um sein Volk auch in der Bibel als "werben" bezeichnet wird7. Kirche muß also demgemäß „ihre Botschaft verkünden entsprechend dem Wort: Gehet hin zu allen Völkern und lehrt sie das Evangelium. (...) Und in der Tat hat die Kirche ja geworben, solange sie besteht"8. Dome, Kirchenmusik, Ornat, Liturgie und sämtliche Symbolik sind für sie per se Werbung für die Kirche, deren lange Tradition sich von den Apostelbriefen, der mittelalterlichen Propaganda fide, bis zur kirchlichen Predigt manifestiert ${ }^{9}$. Oder wie es der werbefreundliche Pastor Holstein formuliert: "Von einer gewissen Seite aus betrachtet ist die Kirche nichts anderes als ein großartiges Werbeinstrument, nämlich eben für die Gedanken des Christentums" ${ }^{\prime 10}$. Letztendlich sind dies aber nur Sprachspiele ${ }^{11}$. Es ist unerheblich, woraus sich der Begriff Werbung etymologisch oder traditionell ableitet und/oder ob Kirche von jeher um Christen "geworben" hat. Die Frage, ob Kirche heute die Mittel der erst in den letzten einhundert Jahren ausdifferenzierten, auf strategischem Marketing basierenden Wirtschaftswerbung ${ }^{12}$ einsetzen darf und soll, ist mit solchen Hinweisen allein jedenfalls noch nicht hinreichend $\mathrm{zu}$ beantworten.

Ebenso führen Argumente der Gegner kirchlicher Werbung in die Irre, die diese aufgrund des Wortes Werbung sofort mit Wirtschaftswerbung, vor allem deren ausgeprägter Negativerscheinung in Form niveauloser, glückversprechender Oberflächenspiegelungen und Penetrationen von Redundantem respektive Irrelevantem, gleichsetzen. Auch dies ist unrichtig, da sich Wirtschaftswerbung und kirchliche Werbung natürlich deutlich unterscheiden, ja (was noch zu zeigen ist) unterscheiden müssen ${ }^{13}$.

7 Gemeinschaftswerk der Evangelischen Publizistik (Hg.), Die werbende Gemeinde. Ein Handbuch, Frankfurt 1983, $7 \mathrm{ff}$.

8 J. Bennemann (Anm. 5), 93.

9 W. Wilken, Die Werbung der Kirche, Berlin 1961, 100-101.

10 Zit. n. Wilken, (Anm.9) 97.

1 Hier erliegen die Autoren oftmals dem Problem des Sprachrealismus bzw. Essentialismus; vgl. zu diesem Problem allgemein Karl R. Popper, Das Elend des Historizismus, Tübingen 51979, 21 ff.

12 D. Reinhardt, Von der Reklame zum Marketing. Geschichte der Wirtschaftswerbung in Deutschland, Berlin 1993, $24 \mathrm{ff.}$

13 M. Schütte, Kirchliche Werbung. Aufgaben. Ziele. Möglichkeiten, Düsseldorf/Wien 1966, 20.

Interessant in diesem Zusammenhang ist auch die Äußerung des Pres- 
Beide Mißverständnisse, das der Gegner und das der Befürworter, begründen sich fälschlicherweise aus einem jeweils begrenzten und eindeutig besetzten semantischen Gehalt des Begriffes Werbung. Dabei ist Werbung, zumindest für den Werbetreibenden, ein offener Begriff, der aus sich heraus noch keine Inkompatibilitäten mit irgendwelchen Themen, auch nicht den kirchlichen, determiniert. Ganz richtig führt deshalb das Gemeinschaftswerk der Evangelischen Publizistik auch in einem Handbuch für kirchliche Werbung aus: "Eine genaue Bestimmung und Abgrenzung der Werbung von anderen Kommunikationsformen gibt es nicht. Deshalb soll ... Werbung all das umschreiben, was mit Offentlichkeitsarbeit, Public Relations, Kommunikation, Kontaktpflege, Mission, vertrauensbildende Maßnahmen, Ernstnehmen der Wünsche und Erwartungen der Mitglieder gemeint ist ${ }^{\mu}{ }^{14}$.

Die Frage ist also eigentlich weniger, ob Kirche werben darf, sondern eher ob sie es mit den Mitteln der kommerziellen Wirtschaftswerbung darf und sollte ${ }^{15}$. Seitens ihrer Mitarbeiter muß sich Kirche mit Einwänden wie "Kirche dürfe kein Aufsehen erregen, Werbung sei der Kirche unwürdig, sie werde durch Werbung herabgezogen auf die Ebene des Geschäftlichen, Profanen; sie werde verweltlicht" ${ }^{4}$ auseinandersetzen ${ }^{16}$. Diese Bedenken und Ängste kirchlicher Mitarbeiter bestehen durchaus zu Recht, sie verlangen beachtet und ernstgenommen zu werden. Aber der Teufel steckt nicht in der Werbung, sondern im Detail, nicht die Sache als solche, sondern das "Wie" hat es in sich. Die Quintessenz einer Tagung der katholischen Kirche in Mülheim, November 1961, war deshalb folgerichtig: "Wenn wir nochmals die Frage stellen: Moderne Werbung für die Kirche? - dann geht es nicht mehr um ein ,Ja' oder ,Nein', sondern nur noch um das ,Wie'. Die Kirche muß werben “ ${ }^{\prime 7}$.

\section{Marketing statt Werbung}

Die Einsicht, daß Kirche Werbung treiben muß, entstand schon zu dieser Zeit - also Anfang der 60er Jahre - aus einem Argumentationsdruck der Kirche gegenüber der Gesellschaft heraus. Probleme, die sich damals erst langsam abzuzeichnen begannen, haben sich in den letzten

sesprechers des Bistums Limburg zu einer von einer Agentur entwickelten Plakatkampagne für die vier Bistümer Limburg, Speyer, Trier und Mainz: Es sei "fatal, in diesem Zusammenhang von Werbung zu sprechen. Es handle sich vielmehr um den Versuch, das kirchliche Profil einer lebendigen Glaubensgemeinschaft mit festen Wertgrundsătzen offensiv darzustellen " (Hervorhebung vom Verfasser; zit. n. New business (1992) Nr. 52-53, 6. Gemeinschaftswerk der Evangelischen Publizistik (Anm. 7), 5.

15 Vgl. auch B. Müller, Kommunikation kirchlicher Organisationen. Funktionen der Werbung in der Kirche, Gütersloh 1975, 17.

J. Bennemann (Anm. 5), 90.

17 M. Schütte (Anm. 13), $16 \mathrm{f}$. 
Jahren deutlich verstärkt. Dümpelten die Kirchenaustritte der beiden großen Volkskirchen Anfang/Mitte der 60er Jahre noch bei ca. 25.000 (katholische Kirche) bzw. ca. 40.000 (evangelische Kirche) pro Jahr herum, stiegen sie Anfang der 70er Jahre deutlich auf rund 75.000 bei den Katholiken und teilweise schon ca. 200.000 bei den Protestanten ${ }^{18}$. 1992 haben fast 200.000 Katholiken und geschätzte 330.000 Protestanten ihre Glaubensgemeinschaft verlassen ${ }^{19}$. Aber nicht nur die Gemeinden dünnten sich ständig aus, auch der Nachwuchs an Gemeindedienern rekrutierte sich immer spärlicher ${ }^{20}$.

Diese "Auflösungstendenz" mag einerseits mit der allgemein wachsenden Skepsis gegenüber Institutionen und Hierarchien ${ }^{21}$, der wachsenden Diskrepanz des "value systems" speziell der jüngeren Generationen gegenüber den Wertesystemen der Kirchen ${ }^{22}$ als auch an trivialeren, z.B. monetären Gründen (Ersparnis von Kirchensteuern) ${ }^{23}$ liegen. Doch diese Gründe sind nicht die eigentlichen, tieferen Ursachen. Auch wenn beispielsweise die Kirchensteuer als wichtigste Begründung für die stetig steigende Zahl der Kirchenaustritte, auch bei den Austretenden selbst, herhalten muB, sieht der ehemalige evangelische Bischof von Berlin-Brandenburg, Martin Kruse, tiefere Wurzeln für den Unmut der Abtrünnigen: "Hätten die Menschen positivere Erfahrungen mit der Kirche, würden sie sich wahrscheinlich nicht an den Abgaben stoßen" 24 .

Die Kirche hat also eindeutige Probleme - mit sich selbst, mit der Öffentlichkeit, aber vor allem mit einer immer breiteren, weiter ausfransenden bzw. abbröckelnden Peripherie ihrer Mitglieder. Daß ein Vertrauensverlust, der über ein "gesundes" kritisches Verhältnis hinausgeht, besteht, ist schwer bestreitbar; und seine Symptome sind kaum von der Hand $\mathrm{zu}$ weisen. Diesen Problemen kann man sich nun entweder stellen und versuchen sie zu bekämpfen, oder man kann den Kopf in den Sand stecken. Die Synode der Evangelischen Kirche in Deutschland attestierte 1992 einem Teil ihrer Mitarbeiter gerade letzteres: "Viele Theologen tun sich schwer damit, den kirchlichen Auftrag im Wettbewerb zu anderen Ideen und Meinungen zu, verkaufen'. Ihre Kritik richtet sich oft auch gegen ökonomisches Effektivitäts-

18 BDW Deutscher Kommunikationsverband ( $\mathrm{Hg}$.), Werbung für den Glauben, Bonn 1980, o.S.

19 M. Merz/S. Rückert, Wer nur den lieben Gott verwaltet, in: Die ZEIT (1994) Nr.7, 13-16, 13.

20 B. Wörndl, Religious Institutions, in: W. Glatzer/K. O. Hondrich/H.-H. Noll/K. Stiehr/dies., Recent Social Trends in Western Germany 1960-1990, Frankfurt 1992, 291.

21 HORIZONT (1993) Nr. 35, 2.

22 B. Wörndl (Anm. 20), 292.

23 S. Piatzer, Verlieren die Deutschen den Glauben?, in: Readers Digest/Das Beste (1993) Nr. 11, 41-49.

24

Ebd., 49. 
denken. Sie würden die Kirche am liebsten vor Wettbewerb schützen. Diese Kritik darf aber nicht dazu führen, daß Kirche auf Werbung um öffentliches Vertrauen verzichtet ... ${ }^{\prime 25}$. Die Probleme, die die Kirche hat und die sich in zunehmenden Kirchenaustritten und einer allgemeinen Säkularisierungstendenz innerhalb unserer Gesellschaft zeigen, können auf keinen Fall allein durch Werbung gelöst werden. Dafür sind sowohl die Probleme als auch ihre Struktur zu komplex.

Vielmehr ist eine globale Strategie notwendig, die erst einmal die gesamte Problematik durchdringt, ihre Ursachen erkundet und dann strategische, umfassende Lösungen erarbeitet. Was not tut ist also ein spezielles (Kirchen)Marketing. Aber auch das Marketing, genauso wie die Werbung, unterliegt schon durch seine Begrifflichkeit gewissen Akzeptanzproblemen innerhalb der Kirchen. „Die für die Führung von Non-profit-Organisationen Verantwortlichen (... kirchliche Beauftragte usw.) sind sich zunehmend der Tatsache bewußt, daß das Marketing auch für sie relevant ist und bei der Lösung der ihnen gestellten Aufgaben nützlich sein könnte. Trotzdem stehen sie dem Marketing etwas skeptisch gegenüber, da diese Wissenschaft den Ruf hat, ausschließlich ein Werkzeug zur Gewinnerzielung zu sein. Die mit dem Begriff Marketing allgemein gedanklich eng verbundenen Begriffe des Verkaufens und Erwerbens sind Themen, mit denen sich Leiter von Non-profit-Organisationen noch nicht recht angefreundet haben" 26 . Auch Tremel lehnt den Begriff Social Marketing wegen seiner „ökonomischen Begründung" ab, da dies "aufgrund des theologischen Verständnisses von Kirche und ihrem Auftrag nicht Basis kirchlicher Öffentlichkeitsarbeit" sein kann ${ }^{27}$. Alternativ schlägt er den Begriff Kommunikationspolitik vor, wobei "Politik" zielgerichtetes, systematisches öffentliches Verhalten bedeuten soll und "Kommunikation" Inhalt und Stil institutioneller Öffentlichkeitsarbeit kennzeichnet ${ }^{28}$.

Ausgehend von der Frage, ob bei dem heutigen Politikverständnis innerhalb der Gesellschaft und der nicht zu allen Zeiten unbedingt als optimal empfundenen Liaison Kirche/Politik der Politikbegriff wirklich so viel glücklicher assoziiert wird, erscheint die Notwendigkeit, den Begriff des Social-Marketing zu meiden, nicht unbedingt einleuchtend, da er sehr wohl mit Kirche bzw. kirchlichen Ansprüchen

25 Gemeinschaftswerk der Evangelischen Publizistik (Hg.), Medien und Kirche. Einführung in das Schwerpunktthema der Synode der Evangelischen Kirche in Deutschland vom 1. bis 6. November 1992 in Suhl, o.O. 1992, 64.

$26 \mathrm{Ph}$. Kotler, Marketing für Nonprofit-Organisationen, Stuttgart 1978, 4.

$27 \mathrm{H}$. Tremel (Anm. 2), 15. Wesentlich pragmatischer und ohne Berührungsängste formuliert $\mathrm{H}$. Michel: "Kirche muß sich nicht mehr entscheiden, $o b$ sie sich mit Sozio-Marketing beschäftigen will. Sie tut es bereits umfangreich." (H. Michel, Kirche und Sozio-Marketing. Überlegungen zu einer ungewohnten Wortverbindung, in: $\mathrm{H}$. Tremel, Öffentlichkeitsarbeit der Kirche, Frankfurt 1984, 27-36, 27)

28 Ebd., 15; vgl. auch H. E. Thormann (Anm. 2), 159-174. 
verträglich ist ${ }^{29}$. Dies gilt zumindest dann, wenn man ihn wie Kotler definiert: „Sozial-Marketing umfaßt den Entwurf, die Durchführung und die Kontrolle von Programmen, die darauf abzielen, das Urteil gewisser Zielgruppen über Ideen oder Praktiken im positiven Sinne zu beeinflussen “ 30 .

Sehr deutlich grenzt er mit drei Punkten das Social-Marketing noch einmal vom erwerbswirtschaftlichen Marketing und dessen ökonomischer Begründung ab:

"1. Erwerbsmarketer versuchen im typischen Falle den identifizierten Bedürfnissen und Wünschen der Zielmärkte entgegenzukommen; Sozial-Marketer versuchen dagegen, die Attitüden oder das Verhalten der Zielmärkte zu verändern.

2. Erwerbsmarketer sehen im typischen Falle ihre Hauptaufgabe in der Gewinnerzielung durch die Förderung der Interessen des Zielmarktes oder der Gesellschaft; Sozial-Marketer fördern die Interessen des Zielmarktes oder der Gesellschaft, ohne dabei einen persönlichen Profit anzustreben.

3. Erwerbsmarketer vermarkten Produkte und Dienstleistungen

29 Der Begriff Marketing ist keinesfalls homogen in seiner Bedeutung, nicht mal innerhalb des engeren Bereichs des Social-Marketings bzw. NonprofitMarketings (vgl. Cl. Holscher, Sozio-Marketing: Grundprobleme und Lösungsansätze zum Marketing sozialer Organisationen, Essen 1977, 29). So versuchen einige Autoren, auch den Begriff des Nonprofit-Marketings weiter auszudifferenzieren und nach Merkmalsausprägungen von Nonprofit-Organisationen unterschiedliche Typen in einer Marketing-Typologie zu verdichten (vgl. hierzu z.B. W. Hasitschka/H. Hruschka, Nonprofit-Marketing, München 1982, 26ff).

$30 \mathrm{Ph}$. Kotler (Anm. 26), 28; m.E. ist aber auch ein ökonomischer Marketingbegriff durchaus mit kirchlichen Zielvorstellungen vereinbar. Marketing läßt sich in dieser Lesart definieren "als zielfunktionales, plandeterminiertes Herbeiführen von Tauschrelationen" (W. Hasitschka/H. Hruschka, Nonprofit-Marketing, München 1982, 10). „Für eine Organisation bedeutet Marketing das wirksame Management der Austauschbeziehungen mit den verschiedenen Märkten und Interessentengruppen" (Kotler, VII). Dies gilt für erwerbswirtschaftliche Unternehmen genauso wie für Nonprofit-Unternehmen, wobei sich allerdings die Oberziele deutlich unterscheiden. Während bei den erwerbswirtschaftlichen Unternehmen das Oberziel das erwirtschaftete Geld ist (und das angebotene Wirtschaftsgut nur Mittel zum Zweck, nämlich Geld ist) verhält es sich bei Nonprofit-Unternehmen genau umgekehrt. Oberziel ist hier das Angebot, die Nutzenstiftung. Die erhaltene Gegenleistung ist hier nur das Mittel zum Zweck, der Möglichkeit des Angebotes der Nutzenstiftung (vgl. Hasitschka/Hruschka, a.a.O., 17-18). Kirche steht oder will mit Menschen (Gläubigen, Gemeindemitgliedern) in einer Tauschrelation stehen. Kirche gibt Lebenshilfe, Beistand, Sinn und Wertsystem und die Gemeindemitglieder bzw. Religionszugehörigen geben die Teilnahme an der Gemeinde Jesu sowie Kirchensteuern und Spendenmittel. 
mit Hilfe von Ideen; Sozial-Marketer vermarkten die Ideen selbst anstelle von Produkten oder Dienstleistungen“31.

Gerade der letzte Punkt ist von besonderer Wichtigkeit. Es soll nämlich nicht, wie so oft von Theologen befürchtet, die Kirche "vermarktet" werden bzw. die Kirche sich selbst "vermarkten", sondern alleine die Idee, die hinter der Kirche steht.

Kirche braucht also ein strategisches Marketing, das einen möglichen Weg beschreibt, die Idee hinter der Kirche wieder zu thematisieren und relevant zu machen. Ein solches Marketing muß auf einem sorgfältig ausgearbeiteten Programm fußen, da die erwünschten Vorgänge in bezug auf Werte- und Verhaltenswandel nicht durch wahllos getroffene Maßnahmen erzielt werden können. Echtes SocialMarketing stellt einen großen Fortschritt gegenüber den üblichen konventionellen Strategien zur Herbeiführung sozialen Wandels dar. Solche hergebrachten "Strategien" antworteten oft nur mit wenig durchdachten, kurzfristigen Werbemaßnahmen, statt strategisch eine Kampagne auf den erforschten Bedürfnissen der Anzusprechenden zu entwickeln ${ }^{32}$.

Allerdings hat dieser Fortschritt noch lange nicht durchgängig in der Werbepraxis Platz gegriffen. Selbst große hochprofessionalisierte Werbeagenturen und intemationale Werbenetworks arbeiten nicht unbedingt nach diesem Standard. Überdeutlich wurde dies anläßlich der Präsentation der aktuellen Kampagne des Evangelischen Stadtkirchenverbandes Köln zu Beginn des Jahres 1993. Von über 130 präsentierenden Werbeagenturen - unter ihnen waren auch die größten und besten Deutschlands vertreten - konnten sich die meisten nicht "vom Glanz und Glimmer üblicher Werbekonzepte lösen"33 und fixierten sich rein auf klassische Werbung. Als eine der wenigen präsentierte eine kleine Frechener Agentur ein strategisches Gesamtkonzept und gewann die Präsentation ${ }^{34}$. Ein anderer Mitbewerber hatte zumindest die Problematik erkannt, als er schrieb: "Wer angesichts dieser Aufgabe (der Werbung für die Kirche) nicht bescheiden wird und anerkennt, daß bisher niemand in unserem Land eine Kommunikationsaufgabe mit diesem Schwierigkeitsgrad zu lösen hatte, hat die Tragweite der Problematik nicht verstanden" 35 .

31 Ebd.

$32 \mathrm{Ph}$. Kotler/E. Roberto, Social Marketing, Düsseldorf/Wien/New York 1991, 39.

33 New business (1993) Nr. 1-2, 5.

34 Textintern (1993) Nr. 1, 8.

35 New business (1992) Nr. 43, 23. 


\section{Die Basis einer Marketingstrategie für Kirche}

Besonders relevant und grundlegend, gerade für eine Kirchen-Marketingstrategie, sind die Punkte Zielgruppensegmentierung, Problemanalyse, Ideenentwicklung und letztendlich, darauf basierend, Kommunikation.

\section{a) Zielgruppensegmentierung}

Am Anfang jeglicher Strategieentwicklung muß geklärt werden, welche Zielgruppen überhaupt angesprochen werden sollen. Im Falle der Kirche bedeutet dies: will Kirche beispielsweise nur die aktiven Gemeindemitglieder oder alle Taufscheinchristen der eigenen Konfession oder alle Taufscheinchristen, also auch die der anderen Konfession oder gar alle, d.h. auch die konfessionell nicht gebundenen Menschen ansprechen? Abhängig von der ausgewählten Zielgruppe müssen auch jeweils deutlich unterschiedliche Konzepte entwickelt werden. Insofern ist die saubere Zielgruppensegmentierung eine unerläßliche Voraussetzung für die korrekte Planung einer Marketingstrategie, die, so scheint es zumindest, bei vielen Kampagnen nicht ausreichend oder höchstens implizit berücksichtigt wird.

\section{b) Problemanalyse und -erforschung}

Die Problemanalyse und -erforschung entspricht im erwerbswirtschaftlichen Bereich des Marketings der Konsumentenforschung, einem relativ weiten Begriff, unter dem sich unterschiedlichste Arten von Forschung und Forschungsansätzen verbergen ${ }^{36}$. Bezogen auf das Thema Kirche sollte Problemanalyse und -erforschung die Eruierung von Einstellungen, Werten und Verhalten in bezug auf Kirche und Religion innerhalb der anzusprechenden Zielgruppe leisten. Besonders wichtig ist es in diesem Zusammenhang $\mathrm{zu}$ erforschen, was je nach vorgenommener Segmentierung die Mitglieder der Kirche entfremdet oder sie austreten läßt oder Nichtkonfessionelle davon fernhält ihr beizutreten oder wieder beizutreten.

\section{c) Ideenentwicklung}

Aus der Zielgruppensegmentierung und den erhobenen Einstellungen, Werten und Verhalten der Zielgruppe heraus müssen Ideen entwickelt werden, wie das Produkt Kirche anziehender, d.h. für die Zielgruppe attraktiver kommuniziert oder sogar gemacht werden kann. Dies könnte durch Marketingmaßnahmen unterschiedlichster Art geschehen. Es können zum Beispiel neue Produkte das Angebot erweitern, verändern oder verbessern. Aber auch eine Überarbeitung des Produktes selbst

36 W. Kroeber-Riel, Konsumentenverhalten, München 51992, 4; G. Behrens, Konsumentenverhalten, Heidelberg 1988. 
oder gar die Schaffung eines neuen Produktes, das ein altes ersetzt, ist theoretisch möglich, wobei letzteres allerdings in diesem speziellen Fall die Gefahr beinhaltet, daß ein neues Produkt nicht mehr Kirche wäre. Jedenfalls müssen alle Marketingmaßnahmen auf einer Zielvorstellung basieren, egal ob sie Erneuerung, restaurative oder fundamentalistische Tendenzen anpeilen. Um nicht mißverstanden zu werden: Es ist klar, „daß die Kirche sich selbst aufgeben würde, wenn ihre Reformbestrebungen einfach um des Erfolges auf dem religiösen Markt Willen geschähen“37.

Was aber durchaus notwendig ist, ist das kritische Hinterfragen der Institution Kirche, wenn man für diese Marketing oder Werbung machen will. Es ist unsinnig, wie auch Wilken ganz richtig von Müller ${ }^{38}$ kritisiert wird, die Augen - aus falsch verstandenem Respekt oder aus der falsch verstandenen Überzeugung, „man könne nur für ein Produkt werben, von dem man hundertprozentig überzeugt ist ${ }^{\prime 39}$ - vor den immanenten Problemen der Kirche zu verschließen. Denn soll "sich die Kirche um ein ,völlig überzeugt sein' von ihrer Sache bemühen, oder soll sie sich darum bemühen, eine überzeugende Sache $\mathrm{zu}$ haben ${ }^{\prime 40}$ ? Unangenehmes, Unpassendes, wirklich Unzeitgemäßes (hier soll keinesfalls einem sich anbiedernden Modernismus das Wort geredet werden) darf nicht vertuscht oder verharmlost werden, Kirche und ihre Berater müssen sich der Krise stellen. "Ob das der Werbewirksamkeit der Kirche förderlich ist, kann unmöglich die primäre Frage sein. Von daher kann sich auch die Erörterung des Themas Kirche und Werbung aus den Fragen der sachlichen Kritik an der Kirche nicht heraushalten, sondern muß sich ihnen stellen. ${ }^{41}$.

\section{d) Kommunikation}

Auf der Basis einer Zielgruppensegmentierung, der Ergebnisse der Konsumentenforschung und den daraus erarbeiteten Ideen muß wiederum eine Kommunikationsstrategie entwickelt werden. Daß Kirche Kommunikation betreiben muß, steht dabei außer Frage.

Ein Grund für die Notwendigkeit von kirchlicher Kommunikation - aber (s.o.) nicht unbedingt von Werbung 42 - liegt in ihrem Auftrag zur Verkündigung. Denn der Kirche "sind der Dienst und das Wort von der Versöhnung aufgetragen. Als ,Leib Christi', als ,Communio sanctorum'

37 B. Müller, Kommunikation kirchlicher Organisationen. Funktionen der Werbung in der Kirche, Gütersloh 1975, 109.

38 Ebd., 103-104.

39 W. Wilken, Praktikum kirchlicher Öffentlichkeitsarbeit, Hamburg 1972, $111 \mathrm{ff}$.

40 B. Müller (Anm. 37), 104.

41 Ebd.

42 Aus dem Verkündigungsauftrag ergibt sich ein Kommunikationsauftrag, aber nicht zwangsläufig auch, wie manchmal fälschlicherweise abgeleitet, ein Auftrag zur Werbung für Kirche (s.o. und vgl. auch Anm. 11). 
ist Kirche ihrem Wesen nach durch Kommunikation bestimmt ${ }^{43}$. (...) Öffentlichkeit gehört zum Wesen der Kirche. Die biblische Botschaft wendet sich an alle Menschen. Gemäß ihrem Auftrag und ihrem Anspruch wirkt die Kirche in der Welt für die Welt. Ihr universales Ziel ist es, allen Menschen zu helfen, daß sie zur Erkenntnis der Wahrheit kommen, zur Erfahrung des in Christus erschienenen und verheißenen Heils"44. Ein weiterer Grund ist die Notwendigkeit, sich innerhalb der Gesellschaft wahrnehmbar zu machen. Denn Gesellschaft und Umwelt werden immer undurchschaubarer. Die moderne Gesellschaft hat sich im Gegensatz zu segmentären Gesellschaften in viele spezialisierte Systeme und Subsysteme funktional ausdifferenziert. Die Kirche erscheint in diesem Kontext nicht mehr als die wichtigste lebensbestimmende Institution neben dem Staat, sondern als eine von vielen konkurrierenden Weltanschauungsgruppen ${ }^{45}$. In dieser Situation kann keine Institution mehr, auch nicht die Kirche, apriori von Aufmerksamkeit ausgehen, sondern jede Institution muß sich im sozialen Aufmerksamkeitskampf ihren individuellen Anteil an der begrenzten Zuteilung erkämpfen. Kirche muß sich wie jede andere Institution wahmehmbar machen neben anderen Institutionen.

Ein weiterer Grund für die Unverzichtbarkeit von Kommunikation liegt neben der Ausdifferenzierung der Gesellschaft in der internen Ausdifferenzierung der Kirche selbst. Kommunikation hat nicht nur bezogen auf Umwelt, sondern auch intern bezogen auf Kirche die Funktion der Reduktion von Umweltkomplexităt. Kirche ist selbst ein stark ausdifferenziertes System und wird vor allem von Nichttheologen als nur schwer durchschaubar erlebt. Dieser Mangel an Transparenz erschwert einerseits die Orientierung innerhalb der Kirche und schürt andererseits unnötigerweise Aversionen aus Un- oder Mißverständnissen. "Die Kirche wird weiterhin im Kreuzfeuer der Öffentlichkeit bleiben. Dagegen ist grundsätzlich auch wenig $\mathrm{zu}$ sagen. Umso wichtiger ist es, daß die Kirchen begreifen, daß man nicht mit vornehmer Nonchalance und gelassenem Nichtstun antworten kann. Die Kirchen müssen vielmehr ihre Aufgabe darin sehen, die Öffentlichkeit auf jede Weise aufzuklären, sie zu informieren, Mißtrauen abzubauen, sich und ihre Arbeit durchsichtig zu machen und um

43 Kirchenamt im Auftrag der Rates der Evangelischen Kirche in Deutschland (Hg.), Die neuen Informations- und Kommunikationstechniken: Chancen, Gefahren, Aufgaben verantwortlicher Gestaltung. Eine Studie der Kammer der Evangelischen Kirche in Deutschland für publizistische Arbeit, Gütersloh $1985,85$.

4 Ebd., 86.

45 Vgl. hierzu auch: N. Luhmann, Öffentliche Meinung, in: ders., Politische Planung. Aufsätze zur Soziologie von Politik und Verwaltung, Opladen 31983, 9-34; sowie Fr. Neidhardt, Jenseits des Palavers. Funktionen politischer Offentlichkeit, in: W. Wunden ( $\mathrm{Hg}$.); Öffentlichkeit und Kommunikationskultur. Beiträge zur Medienethik (Bd. 2), Hamburg/Stuttgart 1994, 19-30, hier bes. 21 . 
Kontakte zu werben" 46 . Kirche und Öffentlichkeit bilden kein Gegenüber, vielmehr muß Kirche ein Teil der Öffentlichkeit sein ${ }^{47}$.

\section{Arten kirchlicher Kommunikation}

Kirchliche Kommunikation ist und muß breit angelegt sein. Sie kann sich nicht auf einzelne Kommunikationsarten beschränken, sondern muß ausgehend von einer allgemeinen gesellschaftlichen Informationsüberflutung, im Sinne integrierter und somit wirksamerer Kommunikation, einer breit und möglichst gesamtheitlich geplanten Strategie folgen ${ }^{48}$. Keinesfalls kann sich Kirche in ihrer Kommunikation - auch nicht in ihrer nach außen gerichteten Kommunikation - allein auf klassische Werbung, verstanden als bezahlte Kommunikation über die klassischen Werbemedien Zeitung, Zeitschrift, Plakat, Fernsehen, Hörfunk und Kino beschränken.

Kirchliche Kommunikation erstreckt sich genauso auf den Gemeinde-Schaukasten, der leider oftmals nur improvisiert und wenig durchdacht konzipiert ist und entsprechend kaum gelesen wird ${ }^{49}$, wie auch den Gemeindebrief, der letztlich auch immer eine Art Werbebrief (direct mailing) ist, den Hausbesuch des Pfarrers, die Pressearbeit, die Handzettel und Besinnungs-Plakate sowie veranstaltete Missionswochen, Pfarraktivitäten (Gruppendiskussionen, Vorträge, Tonbildschauen) und Katholiken- bzw. Kirchentage, die wiederum eine Art ,Eventmarketing' sind ${ }^{50}$.

46 Wilken, a.a.O. (Anm. 39), 11.

47 Kirchenamt im Auftrag der Rates der Evangelischen Kirche in Deutschland, a.a.O., 86.

$48 \mathrm{Vgl}$. M. Bruhn, Effizientes Kommunikationsmanagement - Wandel und Perspektiven im Management der Marketing-Kommunikation, in: Werbeforschung \& Praxis (1993) Nr. 3, 75-86.

49 J. Bennemann (Anm. 8), 110.

50 Wobei die Katholiken- bzw. Kirchentage einen besonderen Stellenwert genießen und inzwischen recht professionell als Medienereignisse kommunikativ ausgewertet werden. „Da dürfen sich die Bischöfe und Oberlaien in den zahllosen Kameras sonnen. Das Medienspektakel wird mittlerweile bis ins Detail mit einer Unzahl von Stäben inszeniert. Kein Journalist bleibt ,unbedient'. Diese kirchlichen Umschlagplätze rangieren für die Medien, was die Quantität der Berichterstattung angeht, kurz nach einem politischen Weltgipfel.“ (B. L. Flöper, Die PR der Kirchen, in: G. Kalt, (Hg.), Öffentlichkeitsarbeit und Werbung. Instrumente, Strategien, Perspektiven, Frankfurt 1989, 97-100, hier: 97) Ein wichtiger, aber als solcher nicht unbedingt anerkannter "Event", der die kommunikative Grundlage eines Dialoges zwischen Kirche und Menschen bilden kann, ist die (feste oder wechselnde, zeitlich begrenzte) Ausstellung von Kunst innerhalb der Kirchen (vgl. dazu auch T. Schierl, Über die Möglichkeit, sich (k)ein Bildnis zu machen, in: St. Agnes/Kath. Bildungswerk Köln (Hg.), Du sollst Dir kein Bildnis machen ..., Köln 1994, 139-143). 
Klassische Werbung kann immer nur eine mehr oder weniger bedeutende Teilrolle im gesamten Kommunikationsmix spielen. Aber keinesfalls braucht kirchliche Kommunikation aufgrund fälschlicher Gleichsetzungen mit Auswüchsen der Wirtschaftswerbung von vornherein ganz auf klassische Werbungen zu verzichten. Im Gegenteil.

\section{Gestaltung kirchlicher Marketing-Kommunikation}

Ob sich Kirche der Werbung bedienen darf oder nicht, kann zwar bei einem neutralen Verständnis des Begriffes Werbung keine (ethische) Frage sein, allerdings welche Formen und Formate von Werbung sie dabei benützen darf, durchaus. Aber abgesehen von ethischen Fragestellungen muß kirchliche Werbung auch in bezug auf ihre erwünschte Wirksamkeit sehr vorsichtig sein, welcher gestalterischen Formate sie sich bedient. Denn noch stärker als bei normaler Markenartikelwerbung dürften bei sowieso gegen Werbung immer stärker sensibilisierten Rezipienten gerade bei falsch oder unbedacht gestalteter kirchlicher Werbung Irritationen und Reaktanz auftreten.

Während Reaktanz vor allem dann auftritt, wenn sich ein Individuum in seinem persönlichen Freiheitsraum bedroht fühlt, z.B. durch sehr deutliche und starke (zumindest so empfundene) Beeinflussungsversuche ${ }^{51}$, entstehen Irritationen durch plattes, aufdringliches Argumentieren, durch Hinweise auf unangenehme Konsequenzen bei Nichtbeachtung und durch penetrante, aufdringliche, peinliche oder geschmacklose Reize ${ }^{52}$. Irritationen wie Reaktanzen vermindern durch die mit ihnen verbundenen Abwehrhaltungen des Rezipienten die Akzeptanz- wie Überzeugungswirkung der Kommunikate deutlich ${ }^{53}$.

Aber neben der wohl eher als gering einzuschätzenden Gefahr, $\mathrm{daß}$ kirchliche Werbung platt und geschmacklos gestaltet wird (dies würde sicherlich gerade bei kirchlicher Kommunikation als besonders diskrepant empfunden werden), muß sich Kirche durchaus vor einer unzeitgemäßen, den Anzusprechenden zu wenig beachtenden selbstreferentiellen Werbegestaltung hüten. Schon Mitte der 60er Jahre wurde allgemein eine mangelnde Aktualität der Kirche in bezug auf das menschliche Leben konstatiert und Änderung empfohlen. Manche Autoren wie z.B. Bennemann glaubten, eine solche Änderung bedeute nur "eine Umformulierung von Gebeten, eine Neuordnung der Liturgie, auch eine neue Deutung des Evangeliums. ... (Das sei) schon notwendig, damit der Christ von heute die Fragestellung seines Lebens im Glauben" wiederfinde ${ }^{54}$. Andere bemerkten die Notwendigkeit einer

51 H. Mayer, Werbepsychologie, Stuttgart 21993, 238ff.

52 W. Kroeber-Riel, Strategie und Technik der Werbung. Verhaltenswissenschaftliche Ansätze, Stuttgart/Berlin/Köln 21990, 129.

53 Ebd.

54 J. Bennemann (Anm. 8), 106. 
tiefergehenden Auseinandersetzung und einer darauf fußenden inhaltlichen Anpassung an die heutige Zeit: Inhalte müssen verständlicher werden. Nicht die äußere Form muß der Zeit angepaßt werden (Jazzmessen, modern aufgemachte Bibeln usw.), sondern die inhaltliche Präsentation, d.h. beispielsweise die Gleichnisse des Neuen Testamentes wären der heutigen Zeit anzupassen. Auch wenn sie zeitlos sind, versteht ein heutiger Industriearbeiter nicht mehr die Gleichnisse vom Winzer, Fischer, Hirten oder Landmann. „Ihr seid das Salz der Erde' wohl das berühmteste Gleichnis - kann ihm nichts sagen, weil Salz heute eine gallenschädliche, nicht mehr seltene Ware zu achtzehn Pfennig ist. Er weiß nichts um den Wert des Salzes im Jahre 30 n.Chr. ${ }^{\circ 5}$. Daß sich in dieser Beziehung leider bis heute noch allzu wenig getan hat, zeigt auch eine aktuelle Kritik an kirchlicher Kommunikation, die zwar von der Verkündigung ausgeht, sich aber auf die gesamte Kommunikation bezieht: "In aller Regel kommt die kirchliche Verkündigung mit Setzungen, mit Dogmatik, eben mit diesen Lehrsätzen, die sie versucht zu interpretieren. Sie interpretiert sie aber nur so, daß sie versucht, die Lehrsätze so zu erhalten, wie sie sind und sie nur in der Sprache unserer Zeit auszudrücken, aber ohne sie der Bilder zu entkleiden, die aus einer Zeit stammen, die längst vergangen ist und deren Bilder mit ihr vergangen sind" 56 .

Kirche muß sich, zumindest nach außen, soweit sie aus ihren "kirchlichen Gettos ausbrechen und in eine nichtchristliche Gesellschaft eindringen " will57, um eine zeitgemäße, zielgruppenangepaßte und nicht belehrende Tonalität in ihrer Ansprache bemühen. Sie muß, dies gilt besonders, wenn sie sich dem Mittel der Werbung bedient, medienadäquat kommunizieren - in ihren Bildern wie in ihren Worten. Das heißt schnell, verständlich und auf den Alltag und seine Problematik bezogen kommunizieren und involvieren. Die Sprache der Kirche hingegen hat in der kirchlichen Journalistik wie in der kirchlichen Werbung oftmals zu wenig Alltagsbezug und wird erst indirekt, über Interpretation verständlich. Das schafft unnötige Distanz. Werbung muß aber gerade Distanzen abbauen und Sympathie schaffen. Dies kann nur mit einer einfachen, sympathischen, alltäglichen und auf die Zielgruppen zugeschnittenen Sprache erreicht werden. Michael Schmolkes auf die Sprache der katholischen Presse bezogene Aussage gilt uneingeschränkt auch für kirchliche Werbung: „Ein Massenmedium ist keine Kanzel und sollte auch von niemandem damit verwechselt werden “ ${ }^{4}$.

M. Schütte (Anm. 17), 97.

56 C. Hondrich, Reklame für Bruder Jesus, in: Cl. Eurich/l. de Haen (Hg.), Hören und Sehen. Die Kirche des Wortes im Zeitalter der Bilder, Stuttgart 1991, 101-112, hier 104.

57 Gemeinschaftswerk der Evangelischen Publizistik, (Anm. 7), 8.

58 M. Schmolke, Sprache in der katholischen Presse, in: Communicatio Socialis (1989) Nr. 1-2, 21-30, 28. 
Auf der anderen Seite muß kirchliche Werbung aber auch zu der Institution Kirche und ihren Mitarbeitern passen. Die Werbung darf keinesfalls etwas vorgaukeln, was die Realität nicht halten kann, denn jegliche externe werbliche Massenkommunikation verursacht i.d.R. auch interne Wirkungen, da das in ihr dargestellte Bild der Kirche mit dem Eigenbild abgeglichen wird. Zu starke Differenzen zwischen diesem kommunizierten Fremdbild und dem Eigenbild führen $\mathrm{zu}$ kognitiven Dissonanzen und können die internen Funktionen der Werbung wie Information, Steuerung und Motivierung erheblich stören bzw. sogar in ihr Gegenteil verkehren. Andererseits dürfen auch Außenstehende nicht getäuscht werden. Interessierte, die durch Vorgaukelung falscher Tatbestände, die Kirche nicht erfüllen kann oder will, veranlaßt werden, sich der Kirche bzw. Gemeinde anzunähern oder sie wieder einmal zu besuchen, werden enttäuscht und somit der Kirche wahrscheinlich endgültig entfremdet. Ebenso unsinnig ist es, durch wesensfremde Aktionen oder aufgesetzte Attraktionen Nachfrage nach Kirche zu stimulieren: „Wer Bibelarbeiten für eine Jungschar anbieten will und mit Tischtennis und anderen Spielen wirbt, hat beides nicht verstanden: zu spielen und Bibelstunden zu halten. Kein Wunder, wenn auf kurz oder lang die junge Schar die Absicht durchschaut und wegbleibt. Mit Speck fängt man Mäuse, diese Redensart darf nicht zum Symbol kirchlicher Einladung werden. Hier werden Enttäuschungen vorprogrammiert, die im negativen Sinn prägend sein können. Der Verlust von Glaubwürdigkeit steht hier auf dem Spiel“"59.

\section{Strategische Alternativen}

Generell kann Kirche zwei Marketingstrategien verfolgen. Die erste wäre eine defensive Strategie, die sich damit zufrieden gibt, den Schrumpfungsproze $B \mathrm{zu}$ stoppen oder wenigstens $\mathrm{zu}$ verlangsamen, d.h. den status quo möglichst zu erhalten. Die zweite Strategie wäre offensiv und würde sich zum Ziel setzen, verlorenes Terrain, also Ausgetretene oder Konfessionslose für die Kirche als möglichst aktive Mitglieder, zurückzugewinnen.

\section{1) Erhaltung des status quo}

Beide Strategien, eine defensive wie auch eine offensive, müssen eine gewisse Öffnung der Kirche erreichen. Denn die "Auflösungstendenz" der Kirchen fuBt nicht nur auf kommunikativen Problemen in Form eines falschen Bildes, das sich die Menschen von der Kirche machen, sondern auch auf realen strukturellen Problemen. So werden durch ein entwickeltes Demokratieverständnis und vielleicht auch durch ein sich weiter durchsetzendes naturwissenschaftlich geprägtes, kritisch-rationales Weltverständnis traditionelle Dogmen immer schwerer akzeptiert.

59 Gemeinschaftswerk der Evangelischen Publizistik, a.a.O. (Anm. 7), 12-13. 
Das soll nicht bedeuten, daß von der Kirche Glaubensgrundsätze erster Ordnung zur Disposition gestellt werden sollen, wodurch die Kirche selbst in Frage gestellt würde. Es ist aber zu fragen, ob nicht dogmatische Festlegungen unterer Ordnung oder sich aus dem kirchlichen Selbstverständnis nicht unbedingt zwingend ergebende Strukturen diskutabel sind. Es geht hier um Probleme, die zumindest teilweise gelöst werden müssen, um einen weiteren Erosionsprozeß zu stoppen. Dazu sind strukturelle Änderungen innerhalb der Kirche notwendig, die aber nur dialogisch zwischen Theologen, Kirchenangestellten und Kirchenmitgliedern ausgehandelt werden können. Dabei sei aber nicht die Gefahr verheimlicht, daß sich eine dem internen Dialog öffnende Kirche wildwüchsig und mit unabsehbarer Richtung verändern kann. Eine Steuerung dieses Prozesses ist nur sehr bedingt möglich ${ }^{60}$.

Eine defensive Strategie, die sich primär an Kirchen- bzw. Gemeindemitglieder richten würde, hätte die Aufgabe, die Mitglieder wieder stärker in das Kirchen- bzw. Gemeindeleben einzubinden, sie zu motivieren, Kirche aktiv mitzugestalten und somit gegen innerlich und äußerlich vollzogene Abwendung zu immunisieren. Eine solche Kampagne startete der Evangelische Stadtkirchenverband Köln im Jahr 1993 unter dem Motto "Misch Dich ein". Entwickelt wurde ein umfassendes Kommunikationskonzept, das zur Aufgabe hatte, ein „innerkirchliches Aufmischen, sprich Bewußtwerden" zu erreichen ${ }^{61}$. Gemeinde, Pfarrer und Kirchenmitglieder, so der Kölner Stadtsuperintendent Manfred Kock, sollten "wieder auf Trab" gebracht werden ${ }^{62}$. Ausdrücklich war diese Kampagne also nicht nur nach außen gerichtet; sie sollte vielmehr intern den Dialog entfachen ${ }^{63}$. Dies ging soweit - und das war Teil des Kommunikationskonzeptes -, daß alle 62 evangelischen Gemeinden in Köln in den Entscheidungs- und Gestaltungsprozeß der Kampagne in Diskussionen miteinbezogen wurden 64 , was allerdings u.a. dazu führte, daß die provokantesten, mutigsten und somit vielleicht gerade die besten Entwürfe wieder verworfen wurden.

Dafür, daß die Kampagne des Evangelischen Stadtkirchenverbandes Köln eine gewisse Wirkung gezeitigt haben könnte, spricht die ausgelöste Aktivierung der Gemeinden, der dankbar aufgenommene Dialog und die scheinbar rückläufige Zahl an Kirchenaustritten im Bereich der Kölner Stadtkirche ${ }^{65}$. Trotzdem kann diese Kampagne, die

60 Zum Steuerungsproblem sozialer Systeme vgl. z.B. H. Willke, Systemtheorie, Stuttgart/Jena 41993, 214ff sowie ders., Systemtheorie entwickelter Gesellschaften, Weinheim/München ${ }^{2} 1993$.

61 Kölner Stadt-Anzeiger vom 19./20. Oktober 1993.

62 Frankfurter Rundschau vom 23. März 1993.

63 Kölner Stadt-Anzeiger vom 23. März 1993.

64 Kölnische Rundschau vom 23. März 1993.

$65 \mathrm{Vgl}$. den Pressespiegel zur Kommunikationskampagne: Evangelischer Stadtkirchenverband Köln/Amt für Presse und Öffentlichkeitsarbeit (Hg.), Misch Dich ein. Pressespiegel. Presse, Rundfunk, Fernsehen. Köln 1994. 
sich neben einer eigenen kampagnenbegleitenden Zeitschrift (die allerdings nur dreimal erschien) in ihrer direkten werblichen Kommunikation auf Großflächenplakate beschränkte, höchstens als eine sog. Teaserkampagne, die nur den eigentlichen Kommunikationsproze $B$ beginnt, verstanden werden ${ }^{66}$. Was nachfolgen müßte, ist eine Kampagne, die den abstrakten Imperativ "Misch Dich ein" thematisch kanalisiert und bindet, damit diese Einmischung nicht um ihrer selbst, sondern um der Kirche bzw. der Kirchenmitglieder Willen begonnen und weitergeführt wird. Die Kampagne hatte auch die Aufgabe, "eine Spirale des Aufbruchs in Gang zu setzen. Nach innen und außen"67. Aber dieser Aufbruch müßte auch weiterhin kommunikativ begleitet und verstärkt werden. Geschieht das nicht, was zu befürchten ist, bleibt die Kampagne ein momentaner Aufschrei, der höchstens kurzfristige Wirkung zeitigen kann, aber schon mittelfristig in Vergessenheit und Bedeutungslosigkeit fallen wird. Sollten sich auch die dialogischen Ansätze, die durch die Kampagne gefördert wurden, zurückbilden, wäre die Enttäuschung der kurzfristig aktivierten Kirchenmitglieder und ihre innerliche oder sogar äußerliche Abwendung vorprogrammiert und es könnte eintreten, was die Gefahr jeder kurzfristigen Kampagne in diesem Bereich ist: Nach anfänglicher Verbesserung der Situation tritt nach einer etwas längeren Zeit letztendlich eine Verschlechterung der Ausgangssituation ein.

\section{2) Expansionsstrategie}

Eine Strategie, die auf die Rückgewinnung oder sogar Ausweitung verlorenen Terrains zielen würde, müßte 1. sehr langfristig angelegt sein und 2 . sehr grundsätzlich kommunizieren.

Schon eine defensive Strategie kann nicht, siehe das Beispiel der Kampagne des Evangelischen Stadtkirchenverbandes Köln, kurzfristig angelegt sein, da für eine derart grundlegende kognitive Veränderung eine gewisse Zeit von Nöten ist. Dies gilt natürlich noch viel mehr für eine Kampagne, die nicht nur auf kognitive Veränderungen, sondern sogar auf Verhaltens- bzw. Werte- und Normveränderungen abzielt. Denn während „einfache“ Kampagnen, die nur durch die Vermittlung neuer Informationen das Bewußtsein für ein wünschenswertes Ziel öffnen wollen und kognitive Veränderungen anstreben, noch relativ leicht ihr Ziel erreichen, ist die Änderung von Verhalten durch eine Kampagne schon eine deutlich schwierigere kommunikative Aufgabe.

66 In einem persönlichen Telefonat mit Günter A. Menne (Presseschef des Evangelischen Stadtkirchenverbandes Köln) am 6.7.1994 konstatierte dieser, daß die Kampagne "Misch Dich ein“ nur eine Teaserkampagne sein könne und auch nur als solche geplant gewesen sei. Eine Nachfolgekampagne des Evangelischen Stadtkirchenverbandes Köln gäbe es aber leider aus verschiedenen Gründen, zumindest in absehbarer Zeit, nicht.

67 Deutsches Allgemeines Sonntagsblatt vom 29. Januar 1993 (Nr. 5). 
Die Änderung fest verwurzelter Wertestrukturen aber ist die schwierigste Aufgabe und hat generell überhaupt nur eine relativ geringe Erfolgsquote $^{68}$.

Bei der Einstellung gegenüber Kirche handelt es sich in der Regel um festverwurzelte Wertestrukturen. Deshalb ist es auch so schwierig, mit Kommunikation diese Einstellungen zu ändern. Eine Werbung wie z.B. die des Kölner Stadtkirchenverbandes muß hier zwangsläufig in ihrer Wirkung sehr begrenzt bleiben. Man kann nicht ein festverwurzeltes Wertebild gegenüber der Kirche mit (immer noch) relativ nichtssagenden Bildern und einem wohlmeinenden, aber in der gesellschaftlichen Kommunikationsüberflutung durch mangelnde (emotionale) Tiefe schnell weggespülten Imperativ "Misch Dich ein“ ändern. Dazu bedarf es weitaus mehr.

Ein weiteres Problem der Kommunikation für Kirche liegt in der mangelnden Transparenz, Offenheit und damit zusammenhängend negativen Thematisierung der Kirche. Wenn über Kirche in nichtkonfessioneller Presse berichtet wird, geschieht dies in der Regel unter eindeutig negativem Vorzeichen 69 ; oder wenn über Kleriker positiv berichtet wird, dann zumeist, wenn sie sich (zumindest vordergründig) kritisch mit Kirche auseinandersetzen 70 . Gegen eine solche schlechte veröffentlichte Meinung zu kommunizieren, ist generell schwierig, noch schwieriger aber ist dies, wenn gegen diese veröffentlichte Meinung ${ }^{71}$ ein Wertewandel in Gang gebracht werden soll. Eine freundliche, beschönigende Kommunikation, die unkritisch Kirche anpreist und Probleme außen vor lassen oder gar bestreiten würde, muß zwangsläufig zu kognitiven Dissonanzen beim Betrachter und bestenfalls im Weiteren zur Nichtbeachtung dieser Kommunikation führen.

Eine offensive Werbung für die Kirche kann also nicht einfach nur eine Werbung für die Kirche, wie die des Kölner Stadtkirchenverbandes, sein. Ein kommunikative Strategie muß hier wesentlich subtiler, umfassender und tiefliegender ansetzen.

Eine strategische Idee könnte beispielsweise sein, sich kommunikativ auf die Kernidee der christlichen Kirchen rückzubesinnen: nämlich auf Jesus Christus. Die historische wie die religiöse Person Jesus Christus übt eine große Faszination aus. Nicht nur auf Christen.

68 Ph. Kotler/ E. Roberto (Anm. 32), 31ff.

69 Vgl. z.B. Lieber Klingeln, in: Der Spiegel (1994) Nr. 26, 38 (o.V.) oder Chr. Wernicke, Brot und Pille, in: Die Zeit (1994) Nr. 26, 3.

70 Vgl. z.B. den Bericht über/bzw. das Interview mit dem Limburger Bischof Franz Kamphaus, in: Die Zeit (1994) Nr. 26, 71 und in: Der Spiegel (1994) Nr. $26,50-55$.

71 Siehe kritisch zum Begriff „Veröffentlichte Meinung": E. G. Mahrenholz, Medien und Macht. Wie sichern wir Pluralismus und Transparenz?, in: W. Wunden (Anm. 45), 43-50, hier 44. 
Jesus ist mediales Thema, das sich unter anderem in der ungeheuren Flut von Publikationen über seine Person deutlich macht ${ }^{72}$. Auch wenn die unterschiedlichen christlichen Konfessionen mehr oder weniger differierende Jesusbilder tradieren, gibt es doch so etwas wie eine Kernidee: ein allgemeines Bild über Jesus und über ein christliches Leben. So grob es in seiner allgemeinen Übereinstimmung sein mag, so grundlegend dürfte es zugleich sein.

Aufgabe einer langfristigen Kampagne für die Kirche wäre es also erst einmal, Jesus, die eigentliche Kernidee der Kirche, allgemein zu thematisieren, bei gläubigen Christen zu aktualisieren und bei jenen, die an höhere Wesen, aber nicht zwingend an den christlichen Gott glauben ${ }^{73}$, diesen diffusen Glauben zu kanalisieren und Jesus als Glaubensgrundlage zum Thema zu machen. Jesus muß wieder kommunikativ in den Mittelpunkt gestellt werden, als eine Person, an die und an dessen Leben man glauben kann, die Lebenshilfe in sich birgt. Das Bedürfnis nach Glauben bzw. Religion ist groß, denn letztendlich gewährt dieser "elementare Beheimatung in einer weitgehend undurchschauten, individuellen Lebenswelt, (...) Orientierung im Angesicht eines komplexen, bedrohlichen Kosmos, (...) Leben in Überlebensmythen"74.

Dieses Glaubensbedürfnis kann von der Kirche aber nur noch bedingt eingelöst werden. Viele Menschen haben sich von der Kirche entfremdet und damit teilweise auch von der Religion, dem christlichen Leben und Jesus selbst. Um die Kirche wieder aktuell zu machen, erscheint es als gangbarer und durchaus erfolgversprechender Weg, sich vor allem in der Kommunikation auf Jesus rückzubesinnen. Denn die Kirche und ihre Dogmatik hat sich von den Menschen entfernt. $\mathrm{Zu}$ dieser Ansicht kommt auch Hondrich, der verlangt, daß Verkündigung sich heute wieder kommunikabel, nachprüfbar und wahrheitsfähig macht und dies durch die Rückbesinnung auf Jesus."Dazu nutzt eine Dogmatik, etwa ein trinitarisches Dogma von Vater, Sohn und Heiligem Geist nichts mehr. Das bedeutet nichts mehr. Das kann nicht mehr nachvollzogen werden. Was aber nutzen kann, ist, wieder zurückzukommen auf Einfaches, Nachvollziehbares, also nicht auf den dogmatisch behaupteten Gottessohn, auf den Menschensohn, auf den Messias, den Kyrios, wie immer die Hoheitstitel in der Bibel lauten, die aus der neutestamentlichen und alttestamentlichen Zeit stammen. Es gilt sie zu

72 R. Thiede, Phantom Jesus, in: Focus (1993) Nr. 51, 124-129.

73 Nach einer aktuellen Umfrage des FORSA-Institutes glauben $66 \%$ der Deutschen an Gott, wovon $63 \%$ an einen christlichen Gott glauben und $34 \%$ an eine Gottheit unabhängig von Religionen. $22 \%$ aller Befragten, die nicht an irgendeien Gott glauben, glauben zumindest an irgendein höhreres Wesen; vgl. Die Woche vom 19.05.1994.

$74 \mathrm{Cl}$. Eurich/I. de Haen, Einleitung, in: dies. (Hg.), Hören und Sehen. Die Kirche des Wortes im Zeitalter der Bilder, Stuttgart 1991, 9-19, hier 14. 
übersetzen in unsere Zeit, in eine Humanität vor dem Tode, in eine Menschlichkeit vor der Auferstehung. Und das bedeutet zum Beispiel, zu erzählen von dem Menschen Jesus, nicht von dem Christus, sondern von dem Menschenbruder Jesus ${ }^{475}$. Jesus ist der Schatz der Kirche. Thn gilt es wieder zu bergen, zu kommunizieren und aktuell zu machen.

Eine solche Kampagne muß emotional sein. Eine emotionale Werbung für die Inhalte des Christentums, die beispielsweise auf die Bedürftigen und Bedürfnisse der Welt, die Zukurzgekommenen, die Geknechteten, die Benachteiligten, das Elend fokussiert. Kirchliche Werbung darf nicht einfach platt sympathisch oder gar anbiedernd sein. Werbung für das Christentum darf oder muß vielleicht sogar unbequem sein. Sie muß mehr als anpreisende, sympathieheischende Werbung sein und somit auch das Unbequeme beinhalten. Denn es gibt ein Bedürfnis, vielmehr ein Verlangen danach, daß auch das Unbequeme ausgesprochen wird, wie auch die Benetton-Werbung zeigt. Aber während bei der Benetton-Kampagne das Problem besteht, daß man einem Produzenten banaler Produkte wie Modeartikel verübelt, mit dem Elend und den Problemen unserer Gesellschaft Aufmerksamkeit für sich und eben diese Produkte zu gewinnen, könnte dies durchaus die Werbung der Kirchen sein ${ }^{76}$. Soziales Engagement wecken und Menschen aktivieren, im Namen des Christentums, den Begriff des Christlichen bzw. des christlichen Lebens wieder positiv aufzuladen. Dies könnten die Aufgaben einer solchen Kampagne sein. Christlichkeit darf nicht als Residuum für verträumte Weltfremde gelten, sondern muß moderne, emotionale, positive Lebenseinstellung werden. (Solche Kampagnen, mit modernen, sympathischen Leuten als Leitbild, die - allerdings nur Kirchenbesuche - als moderne Lebensattitüde vermitteln sollten, haben die amerikanischen Kirchen durchgeführt. Für Kirchenbesuch zu werben, vor allem so, ist natürlich unsinnig. Denn der Kirchenbesuch ist nur Symptom. Niemand wird zur Kirche wieder kommen, weil es chic und zeitgemäß ist, in die Kirche zu gehen. Der Kern der Kirche muß erst wieder "zeitgemäß" werden.)

Erst nach einer längeren Zeit, in der diese Kampagne ohne Absender oder höchstens mit dem Absender "Die christlichen Kirchen" (denn es soll ja gerade erst einmal nicht für die Kirche sondern für Jesus geworben werden) laufen würde, könnte die zweite Stufe dieser Kampagne beginnen, die nach der erreichten kognitiven Veränderung auf eine Verhaltensänderung, nämlich ein christliches Leben, d.h., ein soziales Leben unter Berücksichtigung der christlichen Idee, abzielt. Auch diese Stufe sollte idealerweise noch von den christlichen Kirchen gemeinsam kommuniziert werden. Inwieweit allerdings ein solches Zusammengehen der christlichen Kirchen realistisch ist, sei dahingestellt.

76 Diese Idee verdanke ich Michael Schmolke. 
Als dritte und letzte Stufe dieser Kampagne, die natürlich nur zu einem Teil aus werblicher Kommunikation bestünde und stark durch unterstützende Maßnahmen begleitet würde, müßte dann wiederum nach einer längeren Zeit die Kommunikation auf einer neu erreichten Relevanz des Christentums und des christlichen Lebens aufsetzen und diese Relevanz in einen Bezug zur Kirche bringen, einer Kirche, die sich beispielsweise als Forum und Mittelpunkt aktiv gelebten Christentums begreift.

Es ergibt sich also für die dargestellte Kommunikationsstrategie folgendes strategisches Raster: (s.Abb.)

\begin{tabular}{|lll|}
\hline \multicolumn{3}{|c|}{ Kommunikationsziel soll erreicht werden über Kommunikationsinhalt } \\
1. Stufe kognitive Veränderung & $\longrightarrow$ & $\begin{array}{l}\text { Jesus (als } \\
\text { Identifikationsfigur und } \\
\text { Lebenshilfe) }\end{array}$ \\
2. Stufe Verhaltensänderung & $\longrightarrow$
\end{tabular}

Die aufgezeigte Strategie ist natürlich nicht die einzige Strategie, sondern nur eine von vielen möglichen, wie es allgemein in der kommerziellen Kommunikation niemals nur eine richtige mögliche Strategie, sondern immer mehrere mögliche gibt. Was aber sicherlich für alle Strategien gelten muß, ist die grundlegende Herangehensweise: tiefe, grundlegende strukturelle Probleme, können nur mit einer grundlegenden und umfassenden Strategie gelöst werden.

\section{Fazit}

Die Frage, ob Kirche Werbung treiben darf oder nicht, ist überholt. Kirche muß Werbung machen, wenn sie ihre Probleme innerhalb der Gesellschaft und die Probleme der Gesellschaft gegenüber der Kirche lösen will. Allerdings sind diese Probleme viel zu komplex, als daß sie mit Werbung alleine gelöst werden könnten. Notwendig ist eine umfassende Marketingstrategie, die nicht punktuelle Symptomkuren im Auge hat, sondern, auf einer tiefgreifenden Problemanalyse und Forschung basierend, die Probleme grundsätzlich angeht. Aus dieser Marketingstrategie sollte eine Kommunikationsstrategie abgeleitet werden, die innerhalb eines breiten Kommunikationsmixes zu einem mehr 
oder weniger großen Teil auch klassische Werbung einplanen muß. Egal, wie eine mögliche Strategie kirchlicher Kommunikation aussehen mag, sie muß in jedem Fall langfristig angelegt sein, will sie nicht Gefahr laufen, nur Eintagserfolge zu erzielen. Soll eine Strategie mehr als auf bestenfalls die Erhaltung des jetzigen status quo abzielen, ist es für den Erfolg der Strategie ausschlaggebend, wie tiefgreifend bei der Analyse und später bei den Maßnahmen angesetzt wird. Denn tief verwurzelte Wert- und Normänderungen kann man nicht mit flotten Sprüchen und frommen Wünschen erreichen, sondern nur mit einer tiefgreifenden Strategie und ebenso grundlegenden Maßnahmen.

\section{SUMMARY: Social marketing. A useful way for the Church?}

The question, whether the Church should push forward advertising efforts or not, is old-fashioned. The Church has to advertise, if it wants to resolve its problems within society and the problems of society with the Church. Certainly, these problems are far too complex to be resolved by the use of advertisement only. The necessary changes of values and norms are not possible with fancy talks and pious words, but only with far-reaching strategies and similar fundamental steps.

\section{RÉSUMÉ: Le marketing social. Une voie utile pour l'église?}

La question à savoir si l'église a le droit de faire de la publicité ou non, est dépassée. L'église doit faire de la publicité si elle veut résoudre sés problèmes à l'intérieur de la sociêté et les problèmes de la société face à l'église. Toutefois, ces problèmes sont beaucoup trop complexes pour n'être résolus qu'avec la publicitè. Il est nécessaire d'avoir une stratégie de marketing d'ensemble. Cette stratégie ne doit pas seulement viser au maintient du status quo. Les changements nécessaires de valeurs et de normes ne peuvent pas être réalisés par des paroles légeres et des souhaits pieux, mais seulement par une stratégie profonde et des mesures tout aussi fondamentales.

\section{RESUMEN: Marketing social. ¿Un camino para la iglesia?}

La pregunta de si la iglesia debe o no hacer propaganda es una cuestión superada. La iglesia tiene que hacer propaganda si quiere solucionar sus problemas dentro de la sociedad y los problemas de ésta frente a la iglesia. Sin embargo, estos problemas son demasiado complejos como para lograr su solución solo con propaganda. Necesario es más bien una amplia estrategia de marketing. Esta estrategia no debe pretender sólo la mantención del status quo. Las necesarias transformaciones normativas y de valores no se pueden lograr sólo con lemas atractivos y deseos piadosos, sino solamente con una estrategia profunda y medidas adecuadas. 